Research Paper

\title{
Immunohistochemical biomarkers and volumetric parameters for predicting radiotherapy-based outcomes in patients with p16-negative pharyngeal cancer
}

\author{
Rui-Yun Chen ${ }^{1, *}$, Ying-Chun Lin ${ }^{2,3, *}$, Shang-Wen Chen ${ }^{2,4,5,6}$, Tze-Yi Lin ${ }^{1}$, Te-Chun \\ Hsieh 7,8 , Kuo-Yang Yen ${ }^{7,8}$, Ji-An Liang 2,6 , Shih-Neng Yang ${ }^{2,8}$, Yao-Ching Wang ${ }^{2}$, Ya- \\ Huey Chen ${ }^{9,10}$, Shu-Fen Chiang ${ }^{11}$ and Chia-Hung Kao ${ }^{6,7,12}$ \\ ${ }^{1}$ Department of Pathology, China Medical University Hospital, Taichung, Taiwan \\ ${ }^{2}$ Department of Radiation Oncology, China Medical University Hospital, Taichung, Taiwan \\ ${ }^{3}$ The Ph.D. Program for Cancer Biology and Drug Discovery, China Medical University and Academia Sinica, Taichung, Taiwan \\ ${ }^{4}$ School of Medicine, China Medical University, Taichung, Taiwan \\ ${ }^{5}$ School of Medicine, Taipei Medical University, Taipei, Taiwan \\ ${ }^{6}$ Graduate Institute of Clinical Medical Science, School of Medicine, College of Medicine, China Medical University, Taichung, \\ Taiwan \\ ${ }^{7}$ Department of Nuclear Medicine and PET Center, China Medical University Hospital, Taichung, Taiwan \\ ${ }^{8}$ Department of Biomedical Imaging and Radiological Science, China Medical University, Taichung, Taiwan \\ ${ }^{9}$ Graduate Institute of Cancer Biology, China Medical University, Taichung, Taiwan \\ ${ }^{10}$ Center for Molecular Medicine, China Medical University Hospital, Taichung, Taiwan \\ ${ }^{11}$ Cancer Center, China Medical University Hospital, Taichung, Taiwan \\ ${ }^{12}$ Department of Bioinformatics and Medical Engineering, Asia University, Taichung, Taiwan \\ *Authors contributed equally to this work
}

Correspondence to: Chia-Hung Kao, email: d10040@mail.cmuh.org.tw

Shang-Wen Chen, email: D4634@mail.cmuh.org.tw

Keywords: squamous cell carcinoma, p16-negative pharyngeal cancer, biomarker, positron emission tomography-computed tomography, gross tumor volume

Received: May 07, 2017 Accepted: August 07, $2017 \quad$ Published: August 21, 2017

Copyright: Chen et al. This is an open-access article distributed under the terms of the Creative Commons Attribution License 3.0 (CC BY 3.0), which permits unrestricted use, distribution, and reproduction in any medium, provided the original author and source are credited.

\section{ABSTRACT}

Background: This study determined the prognostic effects of immunohistochemical biomarkers and volumetric parameters predicting radiotherapy-based treatment in patients with p16-negative squamous cell carcinoma of the oropharynx or hypopharynx.

Results: VEGF immunoreactivity $>2$ and GLUT1 overexpression were prognostic factors for lower cause-specific survival. Moreover, both factors were associated with lower disease-free survival. The predictors of lower primary relapse-free survival were VEGF immunoreactivity $>2$ and CT-based gross tumor volume $>16 \mathrm{~mL}$.

Materials and Methods: Immunohistochemical biomarkers in pretreatment biopsy specimens from 60 patients with p16-negative cancer were analyzed using tissue microarrays. Computed tomography (CT)-based and biological tumor volumes were retrieved through fluorodeoxyglucose positron emission tomography-CT. Correlations of cause-specific, disease-free, and primary relapse-free survival with volumetric parameters and the immunohistochemical biomarker score were investigated.

Conclusions: For patients with p16-negative pharyngeal cancer receiving radiotherapy, treatment outcomes can be stratified by VEGF and GLUT1 expression and CT-based gross tumor volume. 


\section{INTRODUCTION}

Human papilloma virus (HPV) infection has become increasingly apparent as a major risk factor for head and neck cancer $[1,2]$. HPV-positive tumors are clinically, pathologically, and etiologically distinct and are more responsive to treatment $[3,4]$. Therefore, despite their occurrence in the same tissue, evidence suggests a biological distinction between HPV-positive and HPVnegative head and neck cancers [5].

The prevalence of human HPV-positive pharyngeal cancer (PC) is lower than $20 \%$ in Asia, which is lower than that in Western countries [6-8]. Although several studies have assessed the ability of various endogenous hypoxic, radioresistant, and proliferative biomarkers to predict treatment outcomes [9-20], few studies have analyzed HPVnegative cancer cohorts. By combining the biological tumor volume determined using fluorine-18 fluorodeoxyglucose (FDG) positron emission tomography-computed tomography (PET-CT) and some immunohistochemical markers, our previous study showed an overexpression of certain endogenous markers associated with increased FDG uptake and treatment outcomes [21]. However, the implementation of precision medicine for HPV-negative PCs remains limited by the lack of thorough information on individual responses to a specific treatment, particularly for patients with advanced stages of cancer. Hence, this study determined the optimal approach for predicting radiotherapy (RT) or chemoradiotherapy (CRT) for organ preservation in patients with HPV-negative PCs by comparing imaging and immunohistochemical studies. The model was based on the assumption that biological and volumetric parameters should be assessed simultaneously to optimize patient selection. In addition, the prognostic effects of biological markers can provide novel therapeutic implications for future clinical trials. The results can facilitate optimizing treatment schemes for HPV-negative patients with high risk factors.

\section{RESULTS}

\section{Treatment outcomes}

Sixty patients were eligible for this study (Table 1 ). At a median follow-up of 23 months (6-72 mo), 27 patients were alive without any observed disease recurrence, and 33 patients had recurrent disease. Twenty-four patients died of tumor recurrence, and 5 died of other malignancies. Supplementary Appendix 1 shows the detailed patterns of treatment failure in the study cohort. In summary, 31 patients remained relapse-free at the primary sites, whereas 29 patients had primary recurrence. Overall, the 2-year CSS, DFS, and PRFS rates were 53\% (95\% confidence interval $[\mathrm{CI}]=41 \%-66 \%), 50 \%(95 \% \mathrm{CI}=38 \%-63 \%)$, and $49 \%$ $(95 \% \mathrm{CI}=37 \%-62 \%)$, respectively. The survival curves according to AJCC T- and N-classification are illustrated in Supplementary Appendix 2.

\section{Comparison of the predictive ability of different threshold methods for local failure}

The ROC curves were analyzed to compare the efficacy of various PET-CT-related parameters and threshold methods for determining the optimal approach for autosegmentation contouring. The results revealed that MTV2.5, TLGp40\%, TLGw40\% were stronger predictors of a residual or recurrent tumor than were other corresponding threshold methods (Supplementary Appendix 3). According to the results, biological tumor volumes determined using the MTV2.5, TLGp $40 \%$, and TLGw $40 \%$ methods combined with GTVp and the optimal cutoff of the immunohistochemistry scoring system for different biomarker expressions (Supplementary Appendix 4) were selected for analyses.

\section{Correlation between volumetric parameters and immunohistochemical biomarker expression}

For all patients, MTV and TLG values were calculated using 4 methods. We failed to obtain comprehensive data on immunohistochemical biomarker expression for 3 patients. GLUT1 overexpression was positively associated with increase of SUVmax and TLG values. VEGF IRS $>2$ was correlated with MTV2.5 and TLGw40\%. c-Met overexpression was correlated with higher MTV2.5 and TLG values (Table 2). Furthermore, higher GTVp was correlated with the overexpression of $H I F-1 \alpha(P=.02, \gamma=0.31), \operatorname{VEGF}(P=.01, \gamma=0.34)$, and $c$-Met $(P=.02, \gamma=0.30)$. Typically, the results revealed weak associations between volumetric data and immunohistochemical biomarkers.

\section{Prognostic factors for CSS, DFS, and PRFS}

As summarized in Table 3, multivariate analyses revealed $V E G F$ IRS $>2[P<.001$, hazard ratio $(\mathrm{HR})=11.21$, $95 \% \mathrm{CI}=4.96-55.98]$ and GLUT1 overexpression $(P<.001$, $\mathrm{HR}=13.51,95 \% \mathrm{CI}=3.67-47.62)$ as prognostic factors for lower CSS. The 2-year CSS rates of patients who had tumors with $V E G F$ immunoreactive score (IRS) $>2$ and $\leq 2$ were $32 \%$ and $66 \%(P<.001)$, respectively, whereas the corresponding rates of patients with $\geq 90 \%$ and $<90 \%$ expression of GLUT1 were $37 \%$ and $75 \%$ ( $P=.01$ Figure 1$)$. Both prognostic factors were also associated with lower DFS $(P<.001, \mathrm{HR}=5.18,95 \% \mathrm{CI}=2.26-11.83$ and $P=$ $.006, \mathrm{HR}=3.21,95 \% \mathrm{CI}=1.40-7.53)$. The 2-year DFS rates of patients who had tumors with $V E G F$ IRS $>2$ and $\leq 2$ were $32 \%$ and $58 \%(P=.03)$, respectively, whereas the corresponding rates of patients with $\geq 90 \%$ and $<90 \%$ expression of GLUT1 were $35 \%$ and $62 \%(P=.03$; Figure 2$)$. Expression of $H I F-1 \alpha \geq 80 \%$ was the third molecular marker of lower CSS $(P=.003, \mathrm{HR}=4.95,95 \% \mathrm{CI}=1.70-14.49)$.

The predictors of lower PRFS were VEGF IRS $>2(P=.02, \mathrm{HR}=2.65,95 \% \mathrm{CI}=1.46-6.10)$ 


\begin{tabular}{|c|c|}
\hline Variables & $N(\%)$ \\
\hline \multicolumn{2}{|l|}{ Sex } \\
\hline Male & 60 \\
\hline Age (year) & range 27 to 78 (median 53 ) \\
\hline \multicolumn{2}{|l|}{ Primary tumor site } \\
\hline oropharynx & $33(55 \%)$ \\
\hline hypopharynx & $7(45 \%)$ \\
\hline \multicolumn{2}{|l|}{ T stage } \\
\hline $\mathrm{T} 1$ & $3(5 \%)$ \\
\hline $\mathrm{T} 2$ & $23(38 \%)$ \\
\hline T3 & $18(30 \%)$ \\
\hline $\mathrm{T} 4$ & $16(27 \%)$ \\
\hline \multicolumn{2}{|l|}{$\mathrm{N}$ stage } \\
\hline $\mathrm{N} 1$ & $8(13 \%)$ \\
\hline $\mathrm{N} 2$ & $48(80 \%)$ \\
\hline $\mathrm{N} 3$ & $4(7 \%)$ \\
\hline \multicolumn{2}{|l|}{ AJCC classification ( 7 th version) } \\
\hline III & $4(7 \%)$ \\
\hline IVA & $51(85 \%)$ \\
\hline IVB & $5(8 \%)$ \\
\hline \multicolumn{2}{|c|}{ Histology grade of squamous cell carcinoma } \\
\hline well differentiated & $20(33 \%)$ \\
\hline moderately differentiated & $17(28 \%)$ \\
\hline poorly differentiated & $10(17 \%)$ \\
\hline unclassified & $13(22 \%)$ \\
\hline \multicolumn{2}{|l|}{ Smoking } \\
\hline smoker & $56(93 \%)$ \\
\hline never-smoker & $4(7 \%)$ \\
\hline \multicolumn{2}{|l|}{ Betel nut quid } \\
\hline yes & $48(80 \%)$ \\
\hline never & $12(20 \%)$ \\
\hline \multicolumn{2}{|l|}{ Alcohol drinking } \\
\hline yes & $43(72 \%)$ \\
\hline never & $17(28 \%)$ \\
\hline Radiation dose (Gy) & median 70 Gy (range, 66 - 74Gy) \\
\hline \multicolumn{2}{|l|}{ Concurrent drug regimen } \\
\hline cisplatin every 3 weeks & $47(78 \%)$ \\
\hline cetuximab & $10(17 \%)$ \\
\hline none & $3(5 \%)$ \\
\hline Median follow-up durations (months) & 23 (range, 6 to 72 ) \\
\hline
\end{tabular}

Abbreviations: AJCC $=$ the American Joint Committee on Cancer.

and GTVp $>16 \mathrm{~mL}(P=.002, \mathrm{HR}=5.52,95 \% \mathrm{CI}=$ 1.85-16.47). The 2-year PRFS rates of patients who had tumors with $V E G F$ IRS $>2$ and $\leq 2$ were $30 \%$ and $56 \%$ $(P=.03)$, respectively, whereas the corresponding rates of patients with GTVp $>16$ and $\leq 16 \mathrm{~mL}$ were $35 \%$ and $64 \%$ $(P=.01$; Figure 3$)$. In addition, no significant difference was observed in PRFS curves between the 2 origin sites of the primary tumors.

To investigate the effects of biological differences on outcomes between the 2 tumor origin sites, subgroup analyses were performed (Supplementary Appendix 5).
$V E G F$ IRS $>2$ remained the major determinant of CSS and DFS after stratification for the tumor origin site. HIF$1 \alpha$ and GTVp expression was a prognostic factor for PRFS in patients with hypopharyngeal cancer.

\section{DISCUSSION}

An understanding of cancer cell phenotypes from genomic expression, immunohistochemical studies, or imaging studies allows oncologists to use individualized therapy. To date, no multigenomic assay is available for 
Table 2: Correlation between PET-CT parameters and protein biomarkers

\begin{tabular}{|c|c|c|c|c|}
\hline Variables & SUVmax & MTV2.5 & TLGp40\% & TLGw40\% \\
\hline \multicolumn{5}{|l|}{ HIF-1 $\alpha$} \\
\hline$P$ value & 0.22 & 0.13 & 0.11 & 0.74 \\
\hline \multicolumn{5}{|l|}{ VEGF } \\
\hline$P$ value & 0.30 & $0.003(\gamma=0.38)$ & 0.06 & $<0.001(\gamma=0.44)$ \\
\hline \multicolumn{5}{|l|}{ GLUT1 } \\
\hline$P$ value & $0.01(\gamma=0.33) 0.12$ & & $0.049(\gamma=0.26)$ & $0.01(\gamma=0.31)$ \\
\hline \multicolumn{5}{|l|}{ CAIX } \\
\hline$P$ value & 0.25 & 0.57 & 0.39 & 0.69 \\
\hline \multicolumn{5}{|c|}{ CLAUDIN-4 } \\
\hline$P$ value & 0.15 & 0.80 & 0.72 & 0.43 \\
\hline \multicolumn{5}{|l|}{ c-Met } \\
\hline$P$ value & 0.10 & $0.02(\gamma=0.31)$ & $0.02(\gamma=0.32)$ & $0.03(\gamma=0.28)$ \\
\hline \multicolumn{5}{|l|}{ Bcl-2 } \\
\hline$P$ value & 0.98 & 0.49 & 0.46 & 0.40 \\
\hline \multicolumn{5}{|l|}{ YAP-1 } \\
\hline$P$ value & 0.57 & 0.43 & 0.95 & 0.81 \\
\hline \multicolumn{5}{|l|}{ Ki-67 } \\
\hline$P$ value & 0.06 & 0.63 & 0.52 & 0.74 \\
\hline \multicolumn{5}{|l|}{ EGFR } \\
\hline$P$ value & 0.053 & 0.62 & 0.30 & 0.78 \\
\hline
\end{tabular}

clinical practice in patients with p16-negative PCs. This study is the first to compare comprehensive quantitative immunohistochemical biomarkers and volumetric parameters for predicting the outcomes in patients with advanced p16-negative PCs receiving definitive RT- based treatment. The assessment of various biomarkers revealed 2 endogenous hypoxic markers, $V E G F$ IRS $>2$ and GLUT1 overexpression, as major prognostic factors for lower CSS and DFS. In particular, VEGF expression maintained its predictive ability per site. In addition,
A

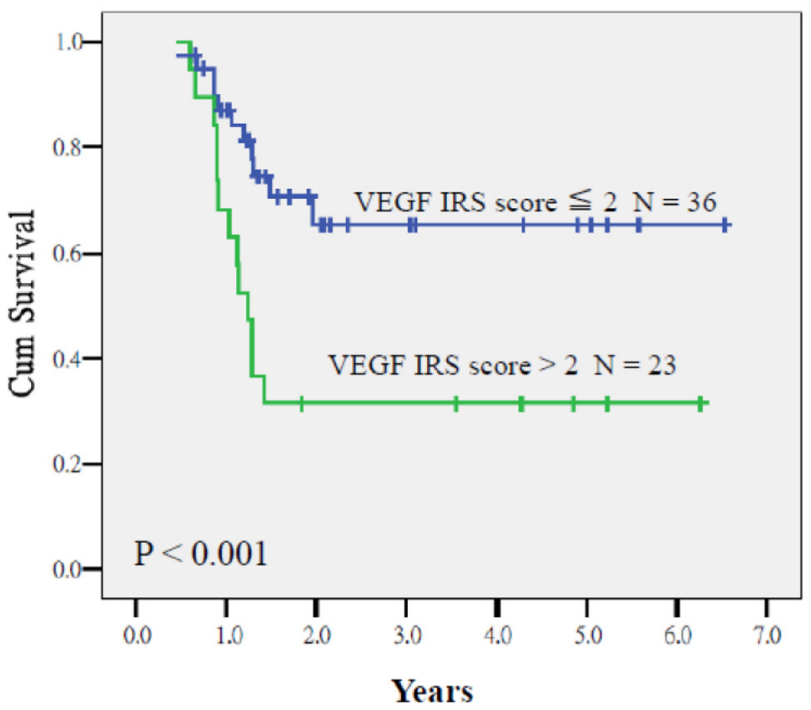

B

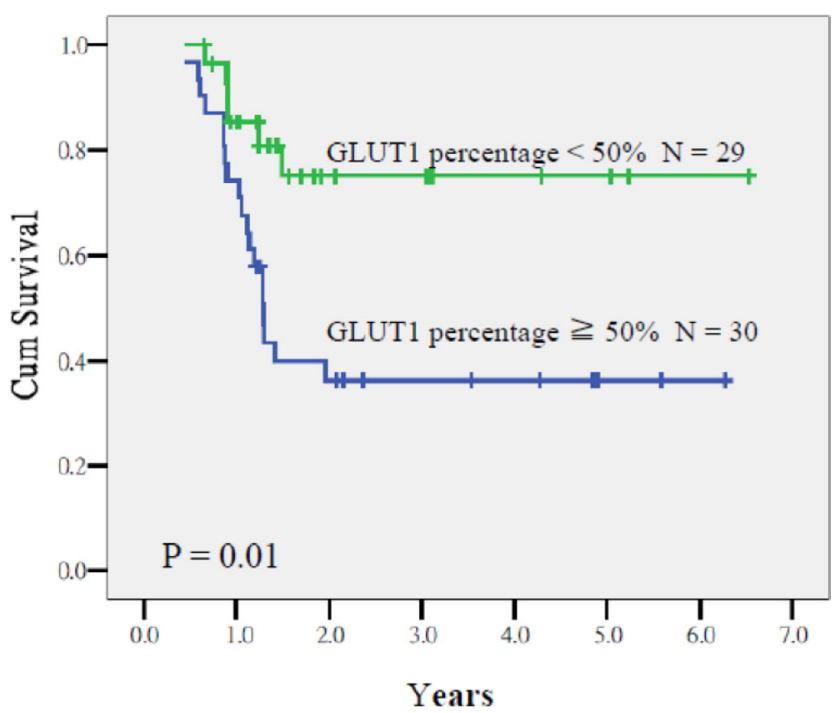

Figure 1: Cause-specific survival of patients who had tumors with $V E G F$ IRS $>2$ and $\leq 2$ (A) and with $\geq 90 \%$ and $<90 \%$ expression of GLUT1 (B) $P<0.001$ and 0.01 , respectively). 
$V E G F$ IRS $>2$ and higher GTVp were predictors of lower local control. On the basis of our finding, certain biological characteristics of tumors might be more determinant than the volumetric parameters alone for RT-based treatment. Moreover, clinical trials with a novel therapeutic strategy should be considered for patients with adverse features.

The most valuable finding is that compared with various biomarkers or volumetric methods, tumor hypoxia remained a major cause of treatment failure in patients with p16-negative PCs because GLUT1, VEGF, and HIF$1 \alpha$ were found to be associated with inferior outcomes. Several studies on head and neck cancers have concluded tumor hypoxia as a major determinant of treatment outcomes [22]. Because endogenous markers can indicate therapeutically relevant levels of hypoxia within tumors, clinical trials assessing the ability of a marker to predict the benefit of specific hypoxia-directed treatment are warranted. Moreover, this study is the first to disclose that GLUT1 overexpression contributed to inferior RT-based outcome in patients with p16-negative PCs. In agreement with a stratified systemic analysis [23], expression status of GLUT1 was associated with unfavorable clinical results of oral squamous cell carcinoma (Odds ratio $=3.79$; $95 \% \mathrm{CI}=1.74-8.24, P=0.0008)$. In addition, our study examined two characters associated with the glycolytic phenotypes of cancers: GLUT1 expression level and FDG uptake on PET-CT. Although GLUT1 expression was positively associated with increased SUVmax and TLG
A

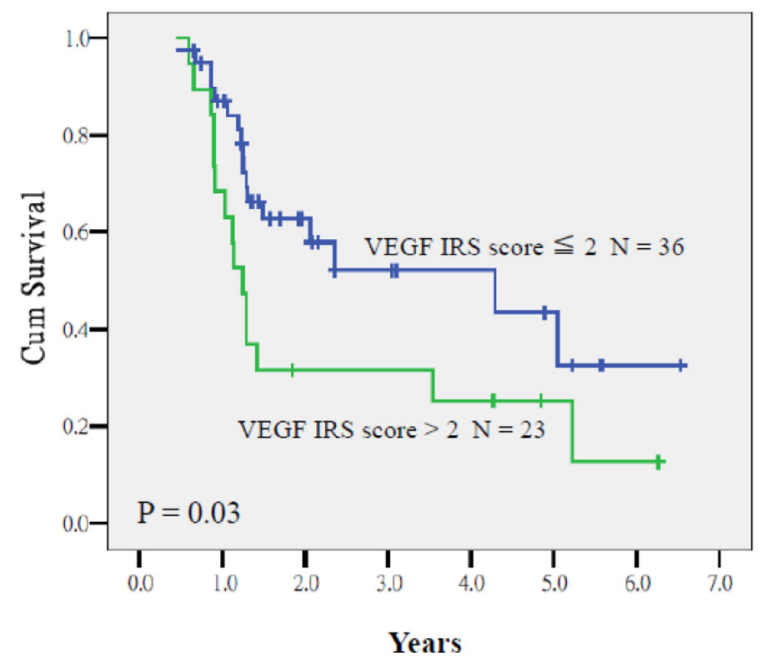

B

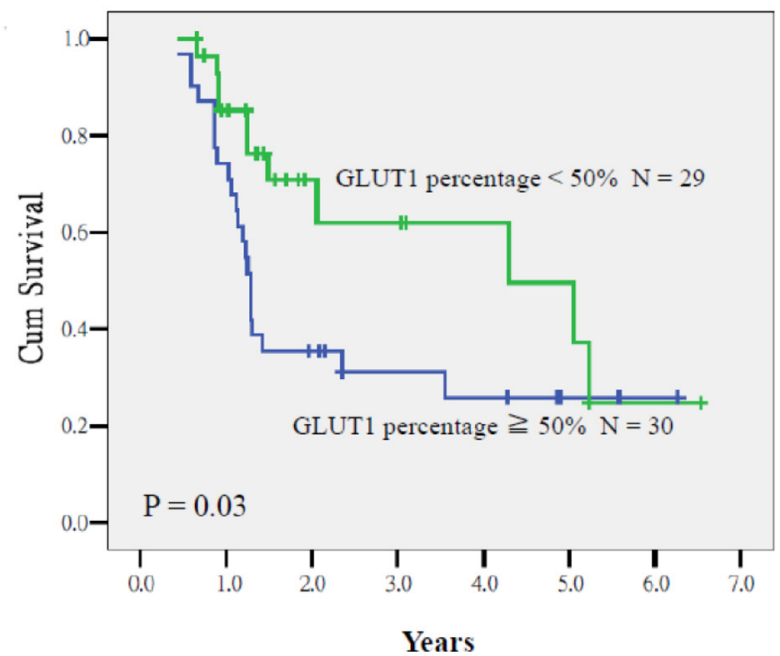

Figure 2: Disease-free survival of patients who had tumors with $V E G F$ IRS $>2$ and $\leq 2$ (A) and with $\geq 90 \%$ and $<90 \%$ expression of GLUT1 (B) $(P=0.03$ and 0.03 , respectively).

A

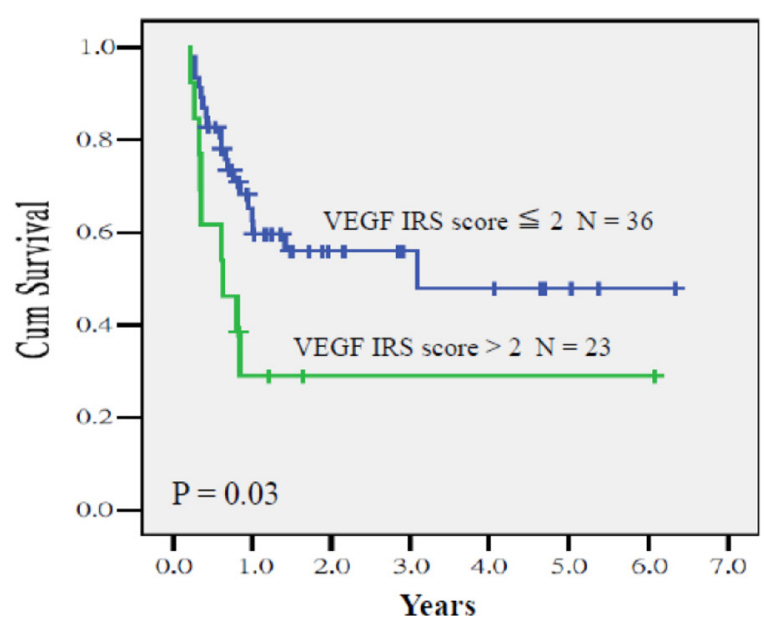

B

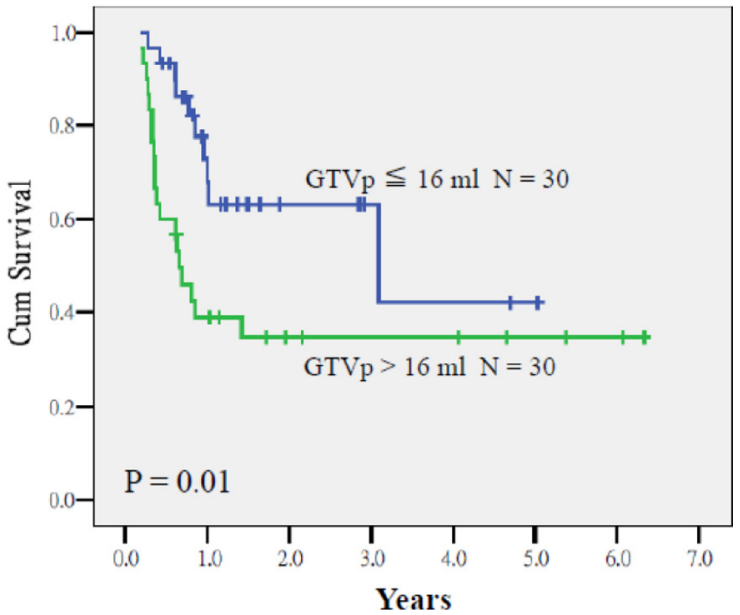

Figure 3: Primary relapse-free survival of patients who had tumors with $V E G F$ IRS $>2$ and $\leq 2$ (A) and GTV $>16$ and $\leq 16 \mathrm{~mL}$ (B) $P=0.03$ and 0.01 , respectively). 
Table 3: Multivariate analysis using the Cox regression model for overall survival, disease-free survival, and primary relapse-free survival among volumetric parameters or protein biomarkers

\begin{tabular}{|c|c|c|c|c|c|c|c|c|c|}
\hline \multirow[t]{2}{*}{ Variables } & \multicolumn{3}{|c|}{ CSS } & \multicolumn{3}{|c|}{ DFS } & \multicolumn{3}{|c|}{ PRFS } \\
\hline & HI & $95 \% \mathrm{CI}$ & $\boldsymbol{P}$ & HP & $95 \%$ CI & $\boldsymbol{P}$ & & IR 95\% CI & $\boldsymbol{P}$ \\
\hline \multicolumn{10}{|l|}{ TNM classification } \\
\hline $\begin{array}{l}\text { T stage } \\
\text { T1-2 vs. T3-4 }\end{array}$ & \multicolumn{8}{|c|}{ T stage } & 0.26 \\
\hline \multicolumn{10}{|l|}{ N-stage } \\
\hline $\begin{array}{l}\text { N1 vs. N2-3 } \\
\text { Primary tumor volume }\end{array}$ & \multicolumn{8}{|c|}{ Primary tumor volume } & \\
\hline $\begin{array}{l}\mathrm{GTVp}(\mathrm{ml}) \\
<16 \text { vs. } \geqq 16 \\
\text { PET-CT parameters }\end{array}$ & 3.70 & $0.83-10.47$ & 0.10 & 3.62 & $0.79-17.35$ & 0.15 & 5.52 & $1.85-16.47$ & 0.002 \\
\hline $\begin{array}{l}\text { SUVmax } \\
<10.3 \text { vs. } \geqq 10.3\end{array}$ & 1.68 & $0.22-13.19$ & 0.62 & 2.30 & $0.25-24.50$ & 0.43 & 2.21 & $0.36-13.70$ & 0.39 \\
\hline \multicolumn{4}{|l|}{ MTV2.5 (ml) } & 9.80 & $0.71-82.18$ & 0.09 & 11.36 & $50.74-86.67$ & 0.08 \\
\hline $\begin{array}{l}\text { TLGp } 40 \% \text { (g) } \\
<62.4 \text { vs. } \geqq 62.4\end{array}$ & \multicolumn{8}{|c|}{ TLGp40\% (g) } & 0.11 \\
\hline $\begin{array}{l}\text { TLGw } 40 \% \text { (g) } \\
<132.5 \text { vs. } \geqq 132.5\end{array}$ & \multicolumn{8}{|c|}{ TLGw40\% (g) } & 0.87 \\
\hline $\begin{array}{l}\text { Primary tumor origin } \\
\text { oropharynx vs. hypopharynx }\end{array}$ & 2.51 & $0.46-12.51$ & 0.29 & 1.66 & $0.34-8.20$ & 0.53 & 2.04 & $0.41-10.42$ & 0.39 \\
\hline \multicolumn{9}{|l|}{ Immunohistochemistry } & \\
\hline $\begin{array}{l}<90 \% \text { vs. } \geq 90 \% \\
V E G F I R S\end{array}$ & \multicolumn{8}{|c|}{ VEGF IRS } & 0.08 \\
\hline$\leqq 2$ vs. $>2$ & 11.21 & $4.96-55.98$ & $<0.001$ & 5.18 & $2.26-11.83$ & 0.001 & 2.65 & $1.46-6.10$ & 0.02 \\
\hline $\begin{array}{l}H I F-1 \alpha \text { stain percentage } \\
<80 \% \text { vs. } \geq 80 \%\end{array}$ & \multicolumn{7}{|c|}{$H I F-1 \alpha$ stain percentage } & $0.56-47.97$ & 0.15 \\
\hline $\begin{array}{l}\text { CAIX stain percentage } \\
\leqq 10 \% \text { vs. }>10 \%\end{array}$ & \multicolumn{8}{|c|}{$C A I X$ stain percentage } & 0.32 \\
\hline $\begin{array}{l}E G F R \text { stain percentage } \\
<65 \% \text { vs. } \geq 65 \%\end{array}$ & \multicolumn{8}{|c|}{$E G F R$ stain percentage } & 0.15 \\
\hline \multicolumn{8}{|l|}{$K i-67$} & $0.68-27.78$ & 0.12 \\
\hline \multicolumn{10}{|l|}{$B c l-2$ stain } \\
\hline $\begin{array}{l}<10 \% \text { vs. } \geq 10 \% \\
C L A U D I N-4 \text { stain percentage }\end{array}$ & 1.12 & $0.11-8.53$ & 0.34 & 1.41 & $0.09-17.64$ & 0.52 & 1.71 & $0.32-9.07$ & 0.53 \\
\hline $\begin{array}{l}\text { score } 0-4 \text { vs. } 5-12 \\
Y A P-1 \text { stain percentage }\end{array}$ & 1.23 & $0.26-5.55$ & 0.80 & 1.82 & $0.48-7.76$ & 0.35 & 1.98 & $0.56-7.01$ & 0.29 \\
\hline $\begin{array}{l}<50 \% \text { vs. } \geq 50 \% \\
\text { c-Met stain intensity }\end{array}$ & 2.47 & $0.44-7.75$ & 0.41 & 2.08 & $0.15-29.2$ & 0.58 & 1.73 & $0.29-10.43$ & 0.55 \\
\hline$\leqq 20 \%$ vs. $>20 \%$ & 1.73 & $0.35-8.64$ & 0.46 & 2.07 & $0.43-15.92$ & 0.27 & 2.05 & $0.78-9.04$ & 0.19 \\
\hline
\end{tabular}

Abbreviations: CSS = cause-specific survival; DFS = disease-free survival; PRFS = primary relapse-free survival; HR = hazard ratio; $\mathrm{CI}=$ confidence interval; GTVp = tumor volume at primary site; SUVmax = maximum standard uptake value; MTV2.5 = pretreatment primary metabolic tumor volume defined by SUV $=2.5 ;$ TLGp $40 \%=$ pretreatment primary lesion glycolysis defined by $40 \%$ of maximal SUV TLGw $40 \%$ : pretreatment primary total body glycolysis defined by $40 \%$ of maximal SUV; IRS = immunoreactive score.

values, GLUT1 overexpression influenced the treatment outcome more straightforward.

Many studies have confirmed the prognostic importance of angiogenesis markers and have found an association of these markers with the progression of tumors and development of lymph node metastases. In accordance with a meta-analysis [16], our study highlighted the prognostic effects of $V E G F$ on RT-based treatment. 
Accordingly, a combination of anti-VEGF target therapies should be used for treating patients with PCs exhibiting $V E G F$ overexpression. Two clinical trials have reported the safety and feasibility of the incorporation of bevacizumab into comprehensive CRT regimens for patients with head and neck cancers $[24,25]$. Further studies must test this regimen in an appropriate subset of patients receiving CRT, particularly those with p16-negative PCs.

In this study, GTVp was the only volumetric parameter affecting local control. GTVp was related to $H I F-1 \alpha, V E G F$, and $c$-Met overexpression. Although we failed to demonstrate the effects of FDG uptake on several endpoints, the PET-related volumetric parameters were clearly associated with certain genomic expression patterns. In particular, the overexpression of GLUT1, $V E G F, H I F-1 \alpha$, and $c$-Met was positively correlated with tumors with an increased FDG uptake, in concordance with previous studies $[26,27]$. The predictive ability of endogenous molecular markers was more satisfactory than that of volumetric parameters. Endogenous biomarkers were the major determinants of the final outcomes; therefore, it is imperative to understand the intrinsic biological characteristics of tumors rather than indiscriminately increasing the RT or drug dose.

The present findings should be cautiously interpreted because of the limited sample size and retrospective design of the study. Therefore, the multivariate analysis per site should be strengthened by conducting additional validation studies by using a large patient cohort. Moreover, RT and CRT outcomes should be assessed with many other biomarkers, such as DNA repair genes, which were not included in the scope of this study. However, the strengths of this study include the uniform treatment strategies and comprehensive quantitative immunohistochemistry methods. Although our findings support the clinical evaluation of hypoxic sensitizers in patients with p16negative locally advanced PCs, a limitation is the lack of ${ }^{18} \mathrm{~F}$-labeled nitroimidazoles PET-CT for imaging the hypoxia for comparison. Because hypoxia presents both treatment challenges and opportunities, characterization is essential to determine the most effective therapy [22]. Considering the more favorable response to conventional RT in patients with p16-positive tumors, the major benefit of hypoxic modification may be improved outcomes in patients with p16-negative tumors [28]. Hence, an appropriate therapy can be decided by evaluating individual tumor biology and assessing established prognostic and predictive biomarkers.

\section{MATERIALS AND METHODS}

\section{Study population}

This retrospective cohort study recruited 92 patients with newly diagnosed squamous cell carcinoma of the oropharynx or hypopharynx who were scheduled to undergo definitive CRT or RT at China Medical University Hospital between January 2007 and December 2013. These patients were selected because they had received RT-based treatment. All patients had received pretreatment PET-CT for RT planning or pretreatment staging; each had a normal serum glucose level before undergoing PETCT. Figure 4 presents the flowchart of patient selection and study design. This study was approved by a local institutional review board (CMUH103-REC2-093FR and DMR99-IRB-010-1).

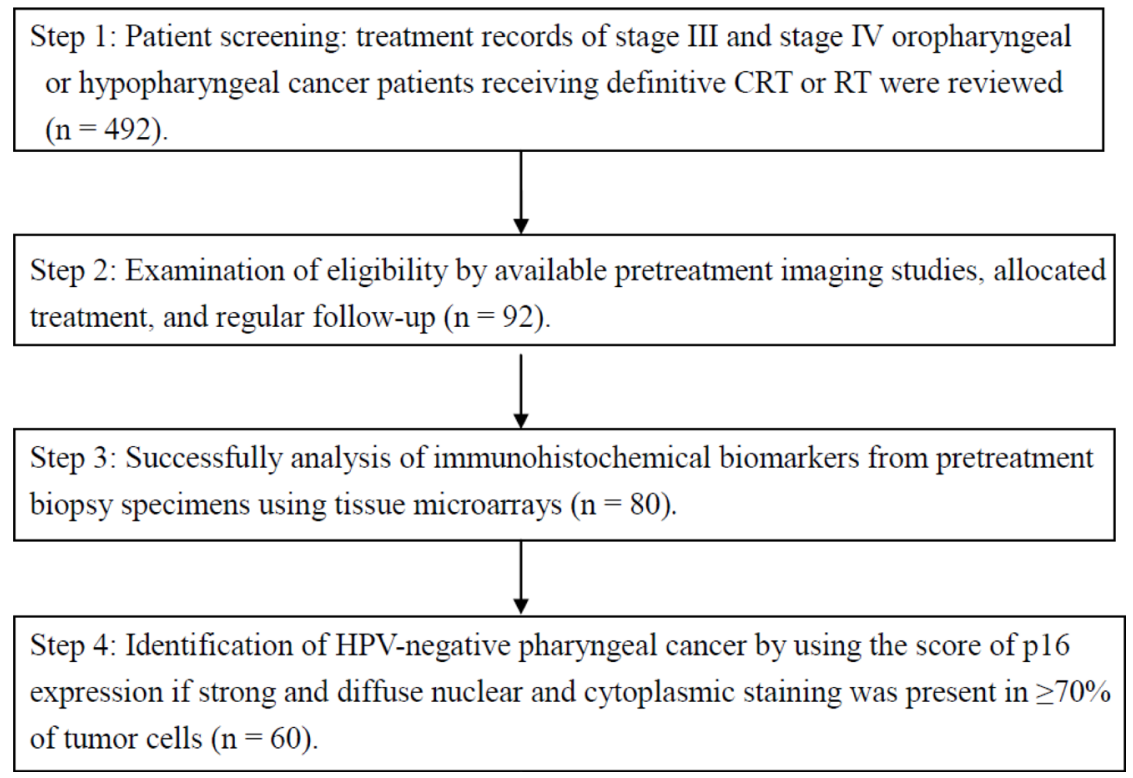

Figure 4: Flowchart of patient selection and study design. 


\section{Immunohistochemistry}

Protein biomarkers in pretreatment incisional biopsy specimens from the primary sites, including endogenous hypoxic markers (GLUT1, CAIX, VEGF, and $H I F-1 \alpha)$ [18], radioresistant biomarkers $(B c l-2$, CLAUDIN-4, YAP-1, and c-Met) [9], a proliferative marker (Ki-67) [20], and a tumor progression factor $(E G F R)[13,19]$, were analyzed using tissue microarrays. Particularly, GLUT1 expression in tumors was reported as a glycolytic phenotype [23, 29], and is transcriptionally activated by hypoxia and $H I F-1 \alpha$ in glucose metabolism [30]. Triplicate $0.6-\mathrm{mm}$ cores were obtained from each paraffin-embedded tumor tissue block. Furthermore, $4-\mu \mathrm{m}$-thick paraffin sections were deparaffinized, microwaved according to standard procedures, and processed for immunohistochemical staining. Among the 92 patients, molecular biomarkers were successfully analyzed in 80 blocks.

The slides were scored by 2 pathologists blinded to clinical outcomes. The staining was graded using the immunoreactive score (IRS) system based on the intensity of staining and percentage of positive tumor cells. Staining intensities of $0,1,2$, and 3 corresponded to negative, mild, moderate, and strong staining; percentages of positive tumor cells were estimated by the observers. The cutoff percentages of the cells for the dichotomization of data were determined for each staining individually by performing receiver operating characteristic (ROC) curve analyses. For HIF-l $\alpha$ and $K i-67$, only nuclear staining was evaluated. Furthermore, for EGFR, CAIX, c-Met, CLAUDIN-4, and GLUT1, only cell membrane staining was evaluated. Both $V E G F$ and $B c l-2$ showed a membranous or cytoplasmic staining pattern. YAP-1 was visualized through cytoplasmic or nuclear staining. Representative images of immunohistochemistry are shown in Supplementary Appendix 6.

\section{HPV status determination by using p16}

In this study, the overexpression of p16 detected through immunohistochemical staining was defined as a surrogate marker of HPV involvement. The expression of p16 was scored as positive in cases of strong and diffuse nuclear and cytoplasmic staining in $\geq 70 \%$ of tumor cells $[4,15]$. Sixty patients $(75 \%)$ were identified as having p16-negative PC; these patients were men with a median age of 51 years. The origin of the tumors was the oropharynx and hypopharynx in 33 and 27 patients, respectively. According to the American Joint Committee on Cancer (AJCC) staging, 4 and 56 had stage III and IV cancers, respectively. Table 1 shows the characteristics of the 60 patients.

\section{PET-CT image acquisition}

All patients were examined using a PET-CT scanner (PET-CT-16 slice, Discovery STE; GE Medical System, Milwaukee, WI, USA) approximately 60 minutes after the administration of $370 \mathrm{MBq}$ of ${ }^{18} \mathrm{~F}-\mathrm{FDG}$; they were required to fast for at least 4 hours before the scan. This procedure provided the standardized uptake value (SUV) of FDG; the maximum SUV (SUVmax) was confirmed by 2 nuclear medicine physicians.

\section{Measurement of metabolic tumor volume and total lesion glycolysis}

The metabolic tumor volume (MTV) and total lesion glycolysis (TLG) were measured from attenuation-corrected FDG-PET images by using an SUV-based automated contouring program (Advantage Workstation Volume Share Version 2, GE Health). This procedure was described in our previous study [28]. The MTV was defined as the sum of the metabolic volumes of the primary tumors. We used an SUVmax of 2.5 (MTV2.5), an SUVmax of 3.0 (MTV3.0), $40 \%$ of SUVmax (MTV40\%), and $50 \%$ of SUVmax (MTV50\%). TLG was determined using the following formula: TLG $=$ meanSUV $\times$ MTV. We used threshold levels equivalent with MTVs: TLG2.5, TLG3.0, TLG40\%, and TLG50\%. Two sets of TLG were determined for each patient: TLGp for the primary tumors and TLGw for the whole body. TLGw was calculated by summing TLGp and all other TLG values of metastatic neck lymph nodes.

\section{Delineation of CT-based tumor volume}

The patients were simulated in an RT setup position on the table of a CT simulator with a head and neck immobilization device. The definition of CT-based tumor volume at the primary site (GTVp) was previously reported [31].

\section{Treatment and follow-up}

RT was performed using a sequential intensitymodulated RT technique [31]. All patients received doses of 1.8 Gy daily and up to a total dose of 68.4-73.8 Gy to the primary tumors or metastatic lymph nodes. The median RT duration was 55 days. Forty-seven patients received concurrent chemotherapy with cisplatin (80$100 \mathrm{mg} / \mathrm{m}^{2}$ on days 1,22 , and 43). Ten patients received combined cetuximab $\left(400 \mathrm{mg} / \mathrm{m}^{2}\right.$ [loading dose] and 250 $\mathrm{mg} / \mathrm{m}^{2}$ ) weekly because of being older than 70 years; 3 patients received RT alone.

After treatment completion, all patients were regularly followed. The definition of local failure was based on the laryngoscopy and neck CT results or both. If 
a patient had a persistent tumor or local recurrence after initial complete remission, salvage surgery was suggested, if technically feasible.

\section{Statistical analyses}

This study used the median GTVp, SUVmax, MTV, and TLG values as cutoff values. To examine the correlations between the parameters and recurrence, ROC curves were constructed to identify the optimal predictive performance among MTVs, TLGs, and the scoring system for different genomic expressions patterns. Correlations between the volumetric and immunohistochemical data were examined using Pearson correlation, with the alpha level set at 0.01 . The study endpoints were cause-specific survival (CSS), disease-free survival (DFS), and primary relapse-free survival (PRFS) rates, which were calculated using the Kaplan-Meier method and log-rank test. Multivariate analysis using Cox regression was performed to examine the effects of explanatory variables on CSS, DFS, and PRFS. Two-tailed tests were used, and $P<.05$ was considered statistically significant. All calculations were performed using SPSS 13.0 for Windows (SPSS Inc, Chicago, IL, USA).

\section{CONCLUSIONS}

For patients with p16-negative advanced PCs requiring definitive RT or CRT, treatment outcomes can be stratified by the immunohistochemical biomarkers of VEGF and GLUT1 and CT-based tumor volume. Further systematic or external validation studies would be required to verify our findings.

\section{Author contributions}

$\mathrm{CH}$ Kao and SW Chen designed the study. RY Chen, YC Lin, SF Chiang, and KY Yen collected the data. RY Chen, YC Lin, and SW Chen performed the statistical analysis, interpreted the data, and drafted the manuscript. SN Yang, JA Liang, ZY Lin, TC Hsieh, YC Wang, and YH Chen provided some intellectual content. SW Chen and $\mathrm{CH}$ Kao approved the final version of the manuscript. All authors have read and approved the final manuscript.

\section{CONFLICTS OF INTEREST}

The authors declare no conflicts of interest.

\section{FUNDING}

This study is supported in part by Taiwan Ministry of Health and Welfare Clinical Trial Center (MOHW106TDU-B-212-113004), China Medical University Hospital, Academia Sinica Taiwan Biobank Stroke Biosignature Project (BM10601010036), Taiwan Clinical Trial
Consortium for Stroke (MOST 106-2321-B-039-005), Tseng-Lien Lin Foundation, Taichung, Taiwan, Taiwan Brain Disease Foundation, Taipei, Taiwan, and Katsuzo and Kiyo AoshimaMemorial Funds, Japan; the Health and Welfare surcharge on tobacco products; and the China Medical University Hospital Cancer Research Center of Excellence (MOHW106-TDU-B-212-144003, Taiwan). The funders had no role in study design, data collection and analysis, publication decision, or manuscript preparation. No additional external funding was received for this study.

\section{REFERENCES}

1. Gillison ML, D’Souza G, Westra W, Sugar E, Xiao W, Begum S, Viscidi R. Distinct risk factor profiles for human papillomavirus type 16-positive and human papillomavirus type 16-negative head and neck cancers. J Natl Cancer Inst. 2008; 100:407-420.

2. Ernster JA, Sciotto CG, O'Brien MM, Finch JL, Robinson LJ, Willson T, Mathews M. Rising incidence of oropharyngeal cancer and the role of oncogenic human papilloma virus. Laryngoscope. 2007; 117:2115-2128.

3. Ragin CCR, Taioli E. Survival of squamous cell carcinoma of the head and neck in relation to human papillomavirus infection: Review and meta-analysis. Int J Cancer. 2007; 121:1813-1820.

4. Ang KK, Harris J, Wheeler R, Weber R, Rosenthal DI, Nguyen-Tân PF, Westra WH, Chung CH, Jordan RC, Lu C, Kim H, Axelrod R, Silverman CC, et al. Human papillomavirus and survival of patients with oropharyngeal cancer. N Engl J Med. 2010; 363:24-35.

5. Masterson L, Moualed D, Liu ZW, Howard JE, Dwivedi RC, Tysome JR, Benson R, Sterling JC, Sudhoff H, Jani P, Goon PK. De-escalation treatment protocols for human papillomavirus-associated oropharyngeal squamous cell carcinoma: A systematic review and meta-analysis of current clinical trials. Eur J Cancer. 2014 ; 50:2636-48.

6. Chien CY, Su CY, Fang FM, Huang HY, Chuang HC, Chen CM, Huang CC. Lower prevalence but favorable survival for human papillomavirus-related squamous cell carcinoma of tonsil in Taiwan. Oral Oncol. 2008; 44: 174- 179 .

7. Li W, Tran N, Lee SC, O'Brien CJ, Tse GM, Scolyer RA, Hong A, Milross C, Yu KH, Rose BR. New evidence for geographic variation in the role of human papillomavirus in tonsillar carcinogenesis. Pathology. 2007; 39: 217-222.

8. Na II, Kang HJ, Cho SY, Koh JS, Lee JK, Lee BC, Lee GH, Lee YS, Yoo HJ, Ryoo BY, Yang SH, Shim YS. EGFR mutations and human papillomavirus in squamous cell carcinoma of tongue and tonsil. Eur J Cancer. 2007; 43:520-6.

9. Akervall J, Nandalur S, Zhang J, Qian CN, Goldstein N, Gyllerup P, Gardinger Y, Alm J, Lorenc K, Nilsson K, Resau J, Wilson G, Teh B. A novel panel of biomarkers predicts 
radioresistance in patients with squamous cell carcinoma of the head and neck. Eur J Cancer. 2014; 50:570-81.

10. Lallemant B, Evrard A, Chambon G, Sabra O, Kacha S, Lallemant JG, Lumbroso S, Brouillet JP. Gene expression profiling in head and neck squamous cell carcinoma: Clinical perspectives. Head Neck. 2010; 32:1712-9.

11. Brockton N, Dort J, Lau H, Hao D, Brar S, Klimowicz A, Petrillo S, Diaz R, Doll C, Magliocco A. High stromal carbonic anhydrase IX expression is associated with decreased survival in P16-negative head-and-neck tumors. Int J Radiat Oncol Biol Phys. 2011; 80:249-57.

12. Vordermark D, Brown JM. Endogenous markers of tumor hypoxia predictors of clinical radiation resistance? Strahlenther Onkol. 2003; 179:801-11.

13. Kumar B, Cordell KG, Lee JS, Worden FP, Prince ME, Tran HH, Wolf GT, Urba SG, Chepeha DB, Teknos TN, Eisbruch A, Tsien CI, Taylor JM, et al. EGFR, p16, HPV titer, Bcl-xL and p53, sex, and smoking as indicators of response to therapy and survival in oropharyngeal cancer. J Clin Oncol. 2008; 26:3128-37

14. Smith BD, Smith GL, Carter D, Sasaki CT, Haffty BG. Prognostic significance of vascular endothelial growth factor protein levels in oral and oropharyngeal squamous cell carcinoma. J Clin Oncol. 2000; 18:2046-2052

15. Gillison ML, Zhang Q, Jordan R, Xiao W, Westra WH, Trotti A, Spencer S, Harris J, Chung CH, Ang KK. Tobacco smoking and increased risk of death and progression for patients with p16-positive and p16-negative oropharyngeal cancer. J Clin Oncol. 30:2102-2111.

16. Kyzas PA, Stefanou D, Batistatou A, Agnantis NJ. Prognostic significance of VEGF immunohistochemical expression andbtumor angiogenesis in head and neck squamous cell carcinoma. J Cancer Res Clin Oncol. 2005; 131:624-630.

17. Kim SJ, Shin HJ, Jung KY, Baek SK, Shin BK, Choi J, Kim BS, Shin SW, Kim YH, Kim JS, Oosterwijk E. Prognostic value of carbonic anhydrase IX and Ki-67 expression in squamous cell carcinoma of the tongue. Jpn J Clin Oncol. 2007; 37:812-819.

18. Klimowicz AC, Bose P, Petrillo SK, Magliocco AM, Dort JC, Brockton NT. The prognostic impact of a combined carbonic anhydrase IX and Ki67 signature in oral squamous cell carcinoma. Br J Cancer. 2013; 109:1859-1886.

19. Hong A, Dobbins T, Lee CS, Jones D, Jackson E, Clark J, Armstrong B, Harnett G, Milross C, O’Brien C, Rose B. Relationships between epidermal growth factor receptor expression and human papillomavirus status as markers of prognosis in oropharyngeal cancer. Eur J Cancer. 2010; 46:2088-96.

20. Couture C, Raybaud-Diogène $H$, Têtu B, Bairati I, Murry D, Allard J, Fortin A. p53 and Ki-67 as markers of radioresistance in head and neck carcinoma. Cancer. 2002; 94:713-22.
21. Lin YC, Chen RY, Chen SW, Hsieh TC, Yen KY, Liang JA, Yang SN, Wang YC, Chen YH, Chow NH, Kao CH. Immunohistochemical studies and fluorodeoxyglucose uptake on positron emission tomography in pharyngeal cancer for predicting radiotherapy-based treatment outcomes. Clin Otolaryngol. 2017; 42:608-619.

22. Brown JM, William WR. Exploiting tumour hypoxia in cancer treatment. Nat Rev Cancer. 2004; 4:437-447.

23. Wang J,Ye C,Chen C,Xiong H, Xie B, Zhou J, Chen Y, Zheng S, Wang L. Glucose transporter GLUT1 expression and clinical outcome in solid tumors: a systematic review and meta-analysis. Oncotarget. 2017; 8:16875-16886. http://doi.org/ 10.18632/oncotarget.15171.

24. Nyflot MJ, Kruser TJ, Traynor AM, Khuntia D, Yang DT, Hartig GK, McCulloch TM, Wiederholt PA, Gentry LR, Hoang T, Jeraj R, Harari PM. Phase 1 trial of bevacizumab with concurrent chemoradiation therapy for squamous cell carcinoma of the head and neck with exploratory functional imaging of tumor hypoxia, proliferation, and perfusion. Int J Radiat Oncol Biol Phys. 2015 ; 91:942-51.

25. Yao M, Galanopoulos N, Lavertu P, Fu P, Gibson M, Argiris A, Rezaee R, Zender C, Wasman J, Machtay M, Savvides P. Phase II study of bevacizumab in combination with docetaxel and radiation in locally advanced squamous cell carcinoma of the head and neck. Head Neck. 2015; 37:1665-71.

26. Yamada T, Uchida M, Kwang-Lee K, Kitamura N, Yoshimura T, Sasabe E, Yamamoto T. Correlation of metabolism/hypoxix markers and fluorodeoxyglucose uptake in oral squamous cell carcinomas. Oral Surg Oral Med Oral Pathol Oral Radiol. 2012 ; 113:464-71.

27. Grönroos TJ, Lehtiö K, Söderström KO, Kronqvist $P$, Laine J, Eskola O, Viljanen T, Grénman R, Solin O, Minn H. Hypoxia, blood flow and metabolism in squamous-cell carcinoma of the head and neck: correlations between multiple immunohistochemical parameters and PET. BMC Cancer. 2014;14:876

28. Lassen P, Eriksen JG, Hamilton-Dutoit S, Tramm T, Alsner J, Overgaard J; Danish Head and Neck Cancer Group (DAHANCA). HPV-associated p16-expression and response to hypoxic modification of radiotherapy in head and neck cancer. Radiother Oncol. 2010; 94:30-35.

29. Kunkel M, Reichert TE, Benz P, Lehr HA, Jeong JH, Wieand S, Bartenstein P, Wagner W, Whiteside TL. Overexpression of Glut-1 and increased glucose metabolism in tumors are associated with a poor prognosis in patients with oral squamous cell carcinoma. Cancer. 2003; 97:1015-1024.

30. Semenza GL. Targeting HIF-1 for cancer therapy. Nat Rev Cancer. 2003; 3:721-732.

31. Chen SW, Hsieh TC, Yen KY, Liang JA, Kao $\mathrm{CH}$. Pretreatment 18F-FDG PET/CT in whole body total lesion glycolysis to predict survival in patients with pharyngeal cancer treated with definitive radiotherapy. Clin Nucl Med. 2014; 39:296-300. 